\title{
Development of a Haptic Device Expressing Hardness of the Virtual Object Using Solenoid
}

\author{
Shenjing Chen", Yuya Kawahara, Akira Yamawaki \\ ${ }^{\mathrm{a}}$ Department of Electrical Engineering and Electronics, Kyushu Institute of Technology, Fukuoka, Japan \\ *Corresponding Author. chen@ boss.ecs.kyutech.ac.jp
}

\begin{abstract}
As one kind of new input device or new information exhibition device, haptic devices representing a tactile feeling of the object are actively researched in recent years. However, existing haptic devices bring some discomfort due to attaching certain devices to fingers, or they need the dedicated exclusive apparatus and panels. Therefore, in this study, we propose a haptic device which can express hardness of the virtual object using solenoid. By simply putting the existing touch panels such as smartphones or tablets on this device, the device can represent a tactile feeling. The device reproduces reaction force to fingers by displacement of the object according to control voltage of the solenoid, and expresses hardness of the virtual object. As a result of the preliminary experiment measuring the reaction force on prototype device, we show that it is possible to control reaction force using solenoid. In addition, for example of application using this device, we design a software keypad that adds the feel of buttons in image displayed on the touch panel. As a result of this case study, compared with the case not changing the hardness of the imaged buttons, we demonstrate that the case changing the hardness by using our device can finish inputting numbers faster and can reduce the miss ratio of the key typing.
\end{abstract}

Keywords: Haptic, Tactile, Solenoid, Control, Sensing.

\section{Introduction}

At present, touch panel equipped electronic devices like tablets, smartphones, and portable game players have been spreading around us. However, operation and information transmission of these devices are depending on eyesight. So, the problems will occur such as eye fatigue, or elderly people or visually impaired people are hard to use touch panel. Therefore, to solve these problems, haptic devices are gathering attention. Haptic devices reproducing the feel of the object have been actively researched in recent years.
As one kind of new input device or new information exhibition device, haptic devices enable person and machine communication through touch. The areas of hepatic devices are also growing rapidly including entertainment, welfare, robotics, psychology, biology, computer science, systems control, and others ${ }^{(1)}$.

However, even though there are constant demands, haptic devices are not very common. This is because existing haptic devices have faults like some discomfort due to attaching certain devices to fingers or difficult to apply in another application because the shape and size of the touch panel is fixed. Therefore, in this study, we propose a haptic device, which needs not attach any devices to fingers, the touch panel can be changed and can express hardness just put smartphones or tablets on this device. We develop a prototype device to investigate whether our device can actually exhibit hardness using solenoid or not. In addition, for example of application using this device, we design a touch panel that adds the feel of buttons on the touch panel's software keypad, and report experimental results of the touch panel.

\section{Proposed System}

\subsection{System organization}

Fig. 1 shows the structure of proposed system. The device consists of a displacement sensor which detects movement of a fixed way, DC solenoids which exhibit hardness and a control unit which controls the displacement sensor and DC solenoids. Smartphones and tablets are put on the fixed way. According to the finger position on the touch panel and the movement of the fixed way, the voltage to DC solenoids is controlled by the pulse width modulation (PWM). The device reproduces reaction force to fingers by displacement of the object according to control voltage of the solenoid, and express hardness of the virtual object. 


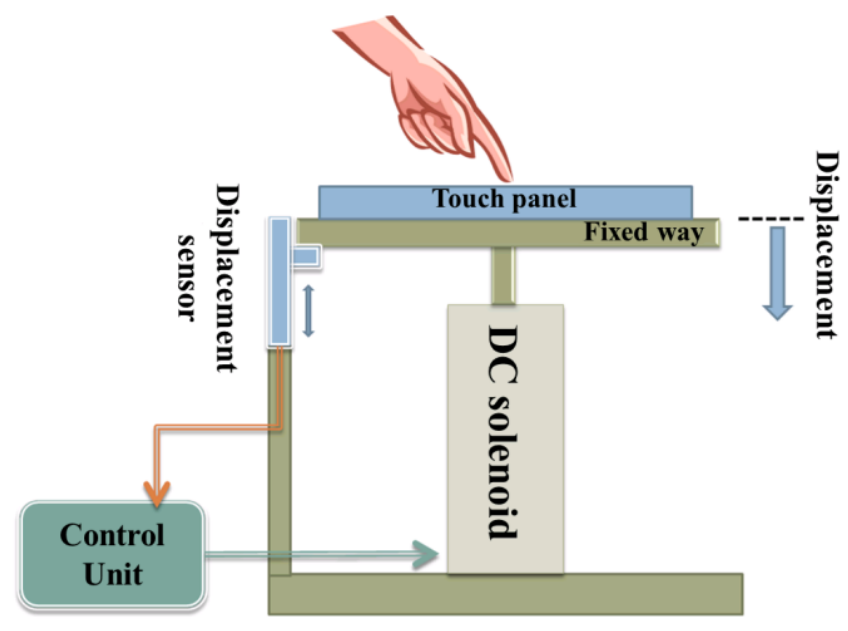

Fig.1. The structure of proposed system

\subsection{Hardness expression using solenoid}

\subsubsection{Structure of solenoid}

Fig. 2 shows the structure of DC solenoid ${ }^{(2)}$. DC solenoid consists of a movable iron core, and coil which generates a magnetic field. When electric current flows through the coil, the movable iron is drawn toward the fixed iron core by magnetism. Since magnetic strength changes by voltage, any force by the magnetic strength can be generated.

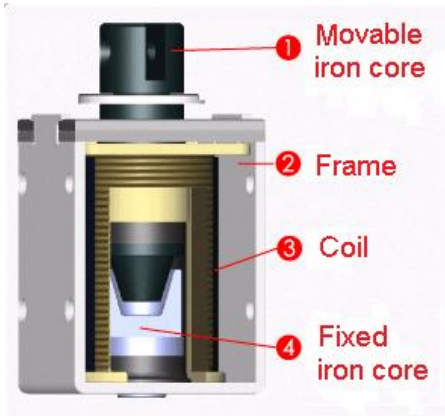

Fig. 2. Structure of solenoid

\subsubsection{Principle of hardness change}

The hardness of a material is expressed by young's modulus $^{(3)}$. It is defined as the ratio of the uniaxial stress over the uniaxial strain. The formula of young's modulus is shown in Equation (1). Definition of young's modulus is shown in Fig. 3.

$$
E=\frac{\frac{F}{S}}{\frac{\Delta x}{X}} \quad \text { (1) } \quad \begin{aligned}
& \text { F: stress } \\
& \text { S: cross section } \\
& \begin{array}{ll}
\triangle \mathrm{x}: \text { strain } \\
\text { X: length }
\end{array}
\end{aligned}
$$

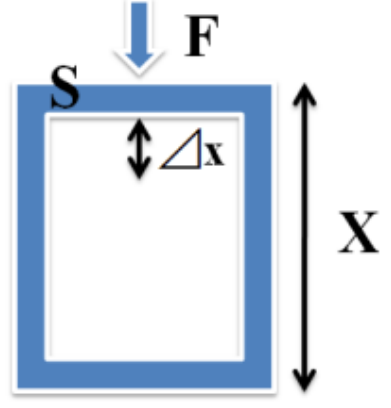

Fig. 3. Definition of young's modulus

In Fig. 3 and Equation (1), $\Delta \mathrm{x} / \mathrm{X}$ expresses uniaxial strain, and $\mathrm{F} / \mathrm{S}$ expresses uniaxial stress.

Next, when solenoid puts strain $\left(\mathrm{F}^{\prime}\right)$ from the bottom, an apparent Young's modulus (E') is shown in Equation (2) and Fig. 4.

$$
\begin{aligned}
& \mathrm{E}^{\prime}=\frac{\frac{\mathrm{f}}{\mathrm{S}}}{\frac{\Delta \mathrm{x}}{\mathrm{X}}}=\frac{\frac{\mathrm{F}+\mathrm{F}^{\prime}}{\mathrm{S}}}{\frac{\Delta \mathrm{x}}{\mathrm{X}}} \\
& =\frac{\frac{\mathrm{F}}{\Delta \mathrm{x}}}{\frac{\mathrm{X}}{\mathrm{X}}}+\frac{\frac{\mathrm{F}^{\prime}}{\mathrm{S}}}{\frac{\Delta \mathrm{x}}{\mathrm{X}}} \\
& =E\left(1+\frac{\mathrm{F}^{\prime}}{\mathrm{F}}\right)
\end{aligned}
$$

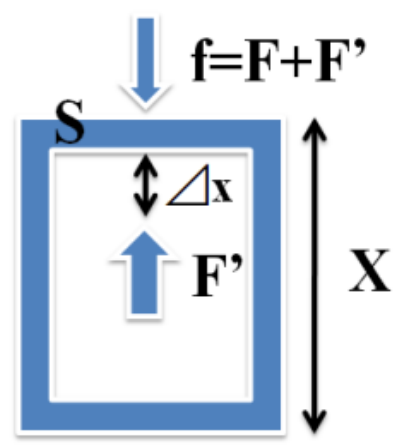

Fig. 4. strain (F') from the bottom

Fig.4 and Equation (2) indicates that the apparent Young's modulus (E') changes in proportion to the strain $\left(F^{\prime}\right)$ from the bottom. So it is possible using solenoid to change the apparent hardness by changing the strain from the bottom. 


\section{Prototype Device}

The block diagram of the prototype device is shown in Fig. 5, and the photographs of prototype device are shown in Fig. 6 and Fig. 7.

There is a fixed way which puts smartphones or tablets on the device, and a displacement sensor and four DC solenoids are put to underneath the fixed way. A displacement sensor and each solenoid are connected to the control unit. The role of each part is shown below.

I. Touch panel such as smart phones, tablets, etc., determine the position of finger touching the screen.

II. PC communicates with the control unit using serial communication, and outputs the image to touch panel.

III. A displacement sensor gets the displacement of the fixed way.

IV. Control unit is composed of three components (Microcomputer, motor driver, and smoothing circuit). Microcomputer which send the measured value of the displacement sensor to touch panel, receive an output value from touch panel, and output PWM signal. Motor driver amplifies PWM signal. Smoothing circuit smooth PWM signal, and output constant voltage.

\section{Touch Panel}

Output the image, Position information

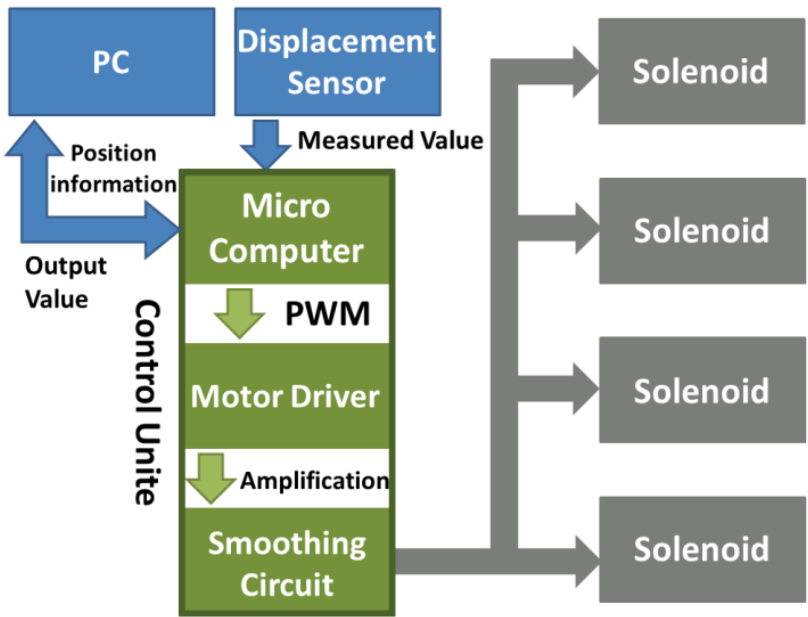

Fig. 5. The block diagram of the prototype device

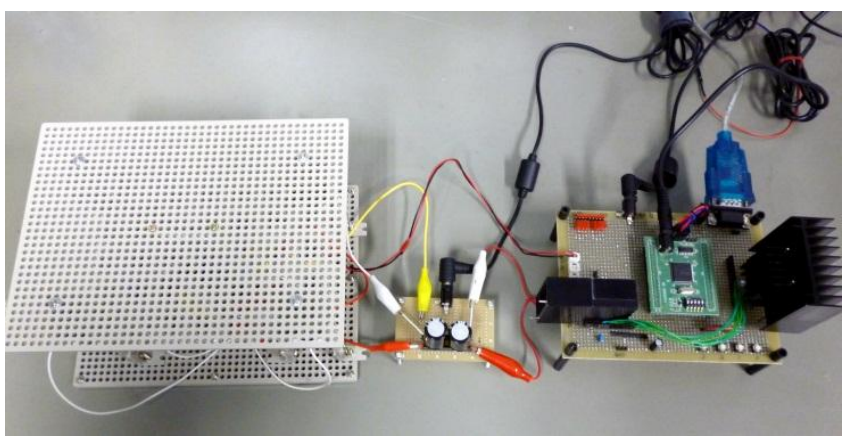

Fig. 6. Prototype device overview

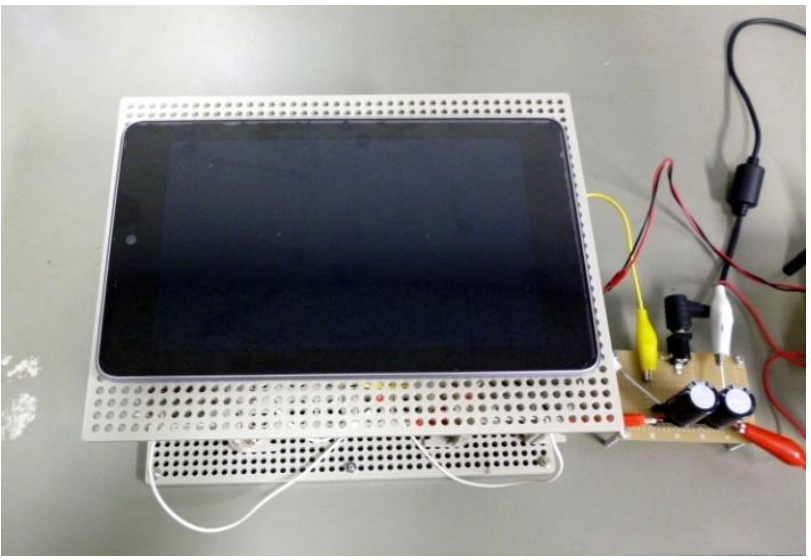

Fig. 7. Tablet on the prototype device

\section{Process Flow}

Fig. 8 shows a typical processing flow of the system when a finger touches the touch panel.

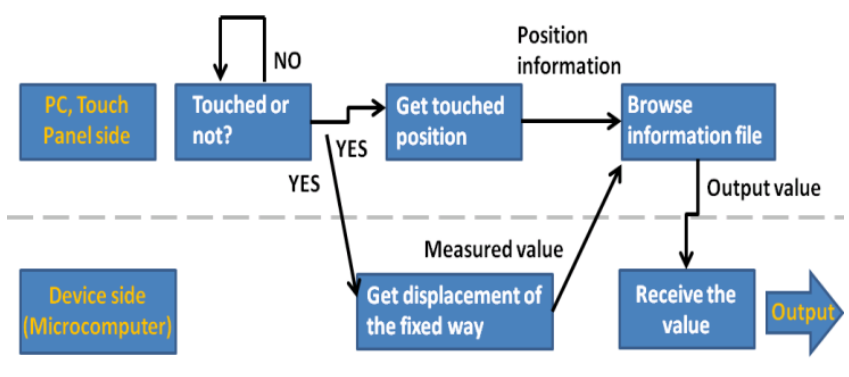

Fig. 8. The process flow

1. Determine whether finger touched the touch panel or not.

2. Determine the position of finger touching the screen. Get the displacement of the fixed way from the displacement sensor.

3. After browsing information file to determine the output value from the position and displacement value, send the output value to the control unit.

4. The control unit receives the output value, and controlling voltage of the solenoid (PWM). 


\section{Preliminary Experiment}

Using the prototype device, we investigate whether reaction force can be actually controlled by solenoid as mentioned in Sect.2.2. This preliminary experiment confirms about the relation between the reaction force and the electric current flowing through solenoid.

\subsection{Reaction force by solenoid using PWM}

The voltage supplied to the solenoid is controlled by the PWM. We measure the relation between the reaction force and the electric current flowing through solenoid when changing the duty ratio of PWM. The experimental results are shown in Fig.9.

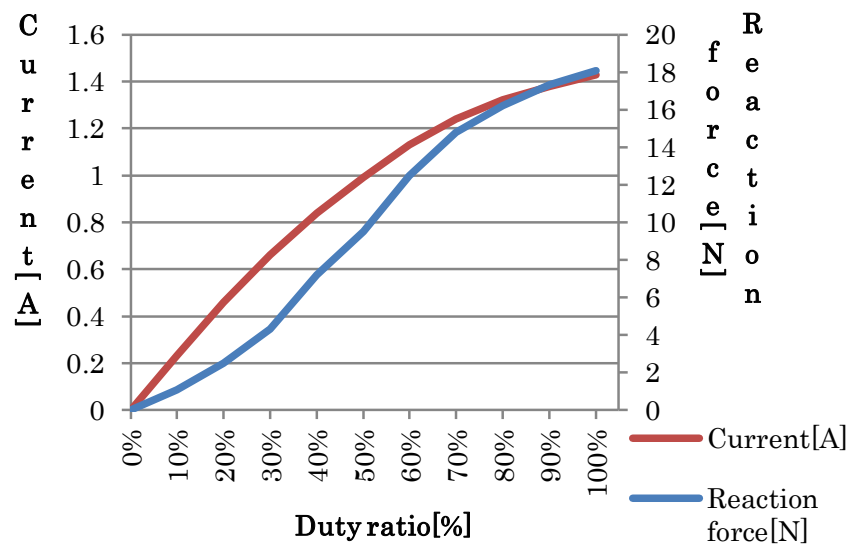

Fig.9. Relation of the reaction force and the current

Fig.9 shows that as the duty ration increases the reaction force becomes stronger. That is, the device becomes harder as mentioned in Sect.2.2. Therefore, hardness can change by duty ratio of PWM.

\section{Case Study}

For example of the application for our device, we have designed a software keypad that is expected to reduce miss typing. The existing touch panel likes smartphones or tablets shown in Fig. 10 are easy to make mistakes. This is because the existing touch panel is not telling whether the button is pushed exactly or not.

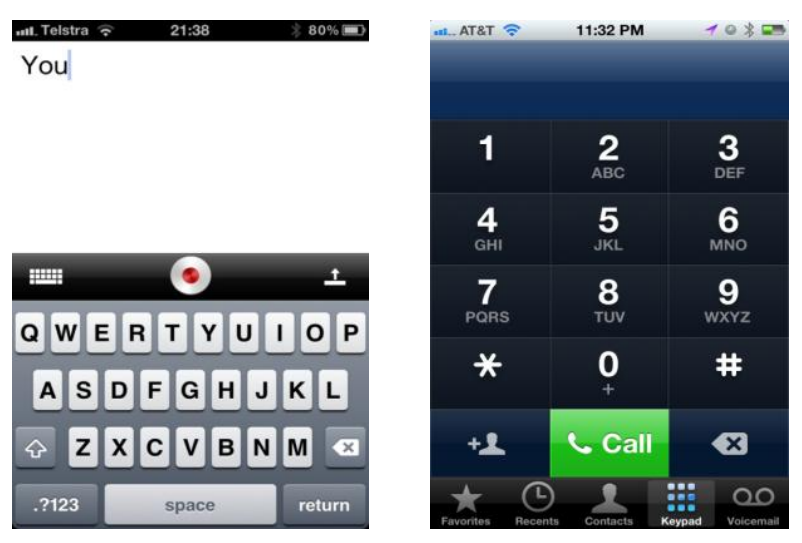

Fig. 10. The existing touch panel

In contrast, our device changes hardness according to the image displayed on screen as follows;

I. Image of button is soft, and can push.

II. Another image is hard, and cannot push.

(Fig. 11)

This software keypad may reduce the miss ratio of key typing by limiting a pushable region physically by changing hardness.

To confirm this effect, we have measured the consumed time for key typing and the number of misses typing by changing hardness and not changing hardness. In addition, we have taken the questionnaire whether subjects can recognize difference in hardness or not. Fig. 12 shows a snapshot of the experiment. The experimental results are shown in Fig. 13, and Fig. 14.

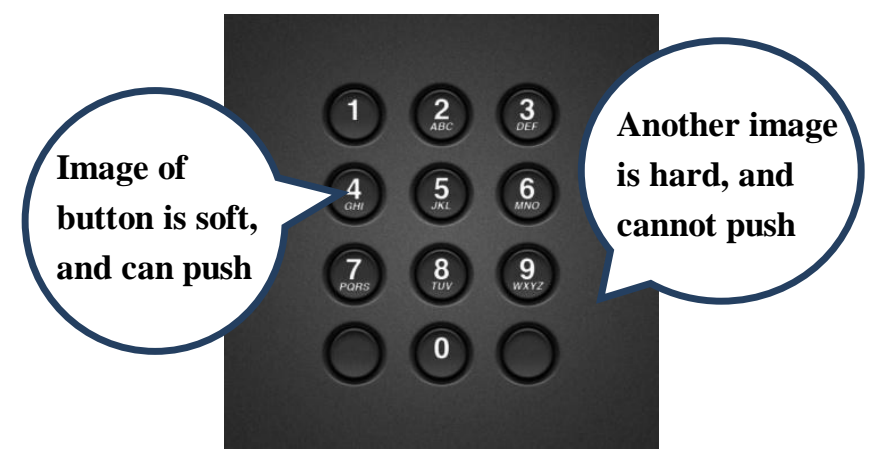

Fig. 11. The image of software keypad

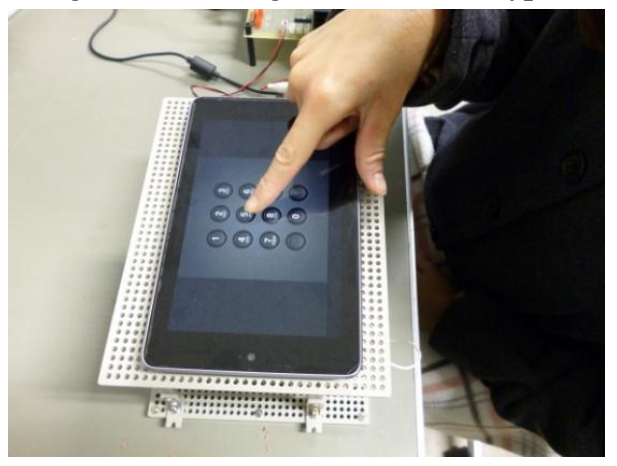

Fig. 12. Snapshot of the experiment 
Input value: Eleven random numbers

Subjects: Six persons (twenties)

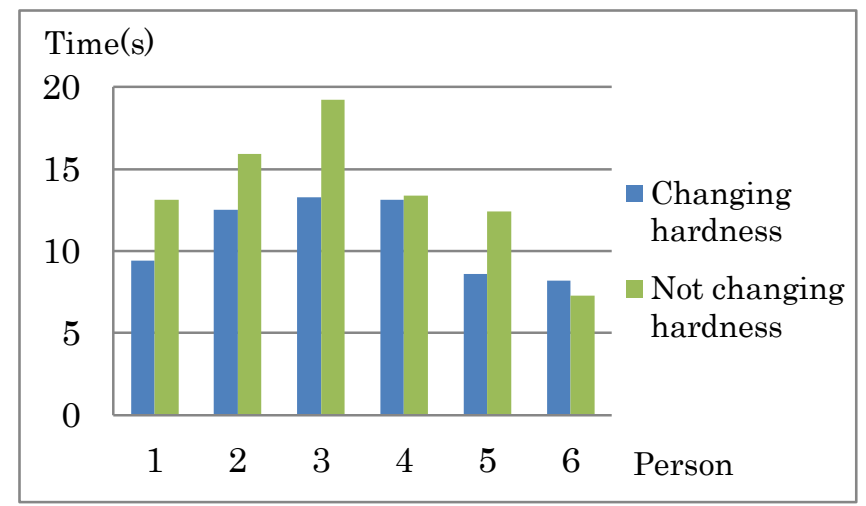

Fig. 13. Consumed time for key typing (individual)

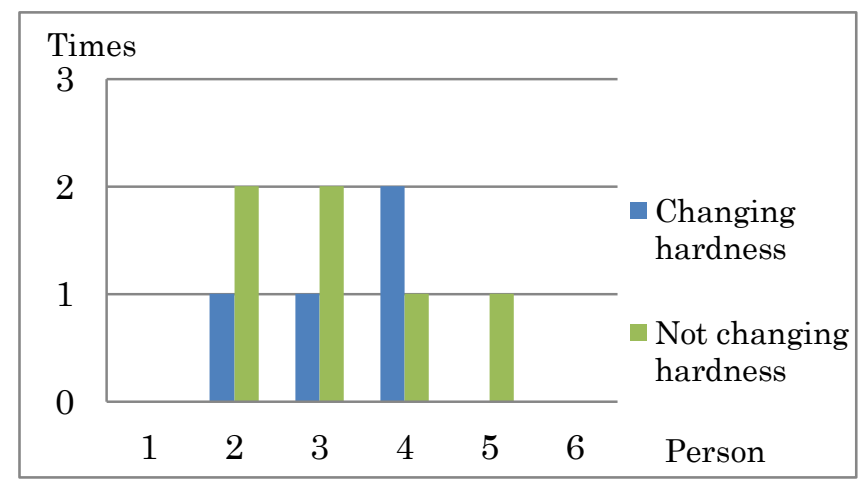

Fig. 14. Number of misses typing (individual)

Questionnaire asks whether the difference of hardness is recognized or not. We let the testes evaluate this questionnaire at five grades of poor as 1 to excellent as 5 .

As a result, the average score becomes 4.5.

Compared with the case not changing the hardness of the imaged buttons, the result indicates that the case changing the hardness by using our device can finish inputting numbers faster and can reduce the miss ratio of the key typing. From the results of questionnaire, almost everyone could recognize difference in hardness.

\section{Conclusions}

In this research, we have proposed a haptic device expressing hardness of the virtual object using solenoid. In the preliminary experiment, we measured the relation between the reaction force and the current for the solenoid used. As results, reaction force can be controlled by using solenoid. In addition, as a case study, we have designed a software keypad that adds the feel of buttons in image on the touch panel's software keypad. As results, compared with the case not changing the hardness of the imaged buttons, we have demonstrated that the case changing the hardness by using our device can finish inputting numbers faster and can reduce the miss ratio of the key typing. Therefore, our device can realize a haptic device using solenoids, which is not necessary to attach any devices to fingers, the touch panel can be changed and can express hardness just put smartphones or tablets on this device.

As future work, we will develop the application devices such as follows;

1. A touch panel which transmit information in tactile.

2. Electronic advertisements with the touch feeling as same as real products.

3. A portable game player that can be played by tactile.

\section{References}

(1) Vincent Hayward, Oliver R . Astley, Manuel Cruz-Hernandez, Danny Grant and Gabriel Robles-De-La-Torre: “Sensor Review” , Volume 24, No. 1, pp. 16-29(2004)

(2) http://www.takaha.co.jp/technological/action.html

(3) Yoshiaki IKEDA and Kinya FUJITA : "Transactions of the Virtual Reality Society of Japan” , Vol.9, No. 2, (2004)

(4) Takaharu Kanai, Yuya Kikukawa, Tatsuhiko Suzuki, Tetsuaki Baba and Kumiko Kushiyama "ACM SIGGRAPH 2011 Emerging Technologies”, Article No. 12 